\title{
Article
}

\section{In Vitro and In Vivo Assessment of a New Workflow for the Acquisition of Mandibular Kinematics Based on Portable Tracking System with Passive Optical Reflective Markers}

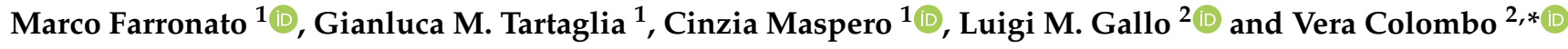 \\ 1 Department of Medicine, Surgery and Dentistry, Fondazione IRCCS Ca' Granda, Ospedale Maggiore \\ Policlinico, University of Milan, 20122 Milan, Italy; marco.farronato@unimi.it (M.F.); \\ gianluca.tartaglia@unimi.it (G.M.T.); cinzia.maspero@policlinico.mi.it (C.M.) \\ 2 Clinic of Masticatory Disorders, Center of Dental Medicine, University of Zurich, 8032 Zurich, Switzerland; \\ Luigi.Gallo@zzm.uzh.ch \\ * Correspondence: vera.colombo@zzm.uzh.ch
}

check for updates

Citation: Farronato, M.; Tartaglia, G.M.; Maspero, C.; Gallo, L.M.; Colombo, V. In Vitro and In Vivo Assessment of a New Workflow for the Acquisition of Mandibular Kinematics Based on Portable Tracking System with Passive Optical Reflective Markers. Appl. Sci. 2021, 11, 3947. https://doi.org/10.3390/ app11093947

Academic Editor: Gabriele Cervino

Received: 26 March 2021

Accepted: 22 April 2021

Published: 27 April 2021

Publisher's Note: MDPI stays neutral with regard to jurisdictional claims in published maps and institutional affiliations.

Copyright: (c) 2021 by the authors. Licensee MDPI, Basel, Switzerland. This article is an open access article distributed under the terms and conditions of the Creative Commons Attribution (CC BY) license (https:/ / creativecommons.org/licenses/by/ $4.0 /)$.

\begin{abstract}
Clinical use of portable optical tracking system in dentistry could improve the analysis of mandibular movements for diagnostic and therapeutic purposes. A new workflow for the acquisition of mandibular kinematics was developed. Reproducibility of measurements was tested in vitro and intra- and inter-rater repeatability were assessed in vivo in healthy volunteers. Prescribed repeated movements $(n=10)$ in three perpendicular directions of the tracking-device coordinate system were performed. Measurement error and coefficient of variation (CV) among repetitions were determined. Mandibular kinematics of maximum opening, left and right laterality, protrusion and retrusion of five healthy subjects were recorded in separate sessions by three different operators. Obtained records were blindly examined by three observers. Intraclass correlation coefficient (ICC) was calculated to estimate inter-rater and intra-rater reliability. Maximum in vitro measurement error was $0.54 \mathrm{~mm}$ and $C V=0.02$. Overall, excellent intra-rater reliability $(\mathrm{ICC}>0.90)$ for each variable, general excellent intra-rater reliability (ICC $=1.00)$ for all variables, and good reliability $($ ICC $>0.75)$ for inter-rater tests were obtained. A lower score was obtained for retrusion with "moderate reliability" (ICC $=0.557)$ in the inter-rater tests. Excellent repeatability and reliability in optical tracking of primary movements were observed using the tested portable tracking device and the developed workflow.
\end{abstract}

Keywords: kinematics; dentistry; mandible

\section{Introduction}

Object identification in a 3D spatial system and precise tracking of movements have always been an issue for non-invasive biomechanical and/or clinical researchers dealing with motion-tracking systems in any field of movement science. Typically, to detect the motion of a body segment, active or passive markers are connected to the skin of the subject and their spatial position is acquired by tracking devices based on different motioncapturing techniques [1]. In the field of dentistry, several systems have been developed in the past with the goal of dynamically analyzing jaw motion [2-5]. In contrast with other body segments, the jaw offered the unique possibility to connect the markers directly to the dental arches, thus excluding the main source of error due to the motion of the skin relative to the bones [6]. For their complex experimental set-up, motion-tracking analysis were originally custom-made devices conceived for laboratory and research purposes. However, technological developments, as downsizing of the hardware and improvement of the acquisition techniques, led to more compact and less-expensive instruments also accessible for clinical set-ups.

A newly commercially available portable system for optical motion-tracking named Polaris Spectra has been developed in the past years by Northern Digital Inc. (Waterloo 
University Canada) and has been successfully used in several medical fields $[7,8]$, showing promising results. This device has the advantage of small size and simple set-up requirements. Studies using this system were conducted in neurosurgery, spinal surgery, robotic surgery, and facial transplantation [7] with a distance error of $0.35 \mathrm{~mm}$ RMS (root mean square- $85 \%$ of cases) [8].

This type of device may be also introduced in a clinical context in the field of dentistry to quantitatively analyze the range of motion of the mandible. In fact, non-invasive tracking systems can detect irregularities in the mandibular function that can otherwise appear clinically normal. For instance, in the case of mandibular movements with a normal mouth opening, a tracking system can identify a change of the mandibular movements during mouth opening into their rotatory (lower temporo-mandibular (TMJ) compartment) and translatory elements (upper TMJ compartment) [9,10]. Furthermore, for a more sophisticated biomechanical analysis of the mandibular motion, a three-dimensional animating technique called dynamic stereometry, has been developed in the past based on active markers, optoelectronic motion-tracking and subject-specific anatomical information obtained utilizing standard tomographic methods (e.g., CBCT, MRI). This method allows the dynamic representation of the relative movement of the mandible with respect to the head and performs quantitative measurements within the joint of the analyzed subject. In particular, the intra-articular space can be quantified and any anatomical point on the articular surface of the joint during functional movements can be tracked [11]. The combination of motion and anatomy, until now confined mainly within the research field, could allow innovative dynamic diagnostic tests applicable for example in DC-TMD nosological classification when translated into the clinical settings [12]. For this reason, a precise and reliable determination of the mandibular motion relative to the head has to be provided by the selected tracking system. Effectively tracked motion of mandibular positioning and movements in 3D could allow precise recreation of its digital dynamic model. Therefore, its accuracy is crucial for the evaluation of the device's effectiveness.

Several authors have proposed methods of mandibular movement registration by the use of a binocular high-precision triaxial electronic translator [13,14], photogrammetry [15], the combination of a digital caliper rule and 3D ultrasonic Jaw Motion Analyzer [16] or by the use of an ultrasound system [17]. Moreover, the registration process allows for the 3D spatial movement record to be combined with 3D-rendered models of the cranio-facial structures obtained from radiological images (CBCT or MRI) including the temporomandibular skeletal structures [18]. The new augmented reality-based systems that are rising in their popularity could in the future largely benefit from optical trackers since they can be used as the link between the digital and real positions of an object in a $3 \mathrm{D}$ system [19]. The precision of the transferred radiological reconstruction to the tracking system depends on the complete workflow of the experiment: precision of the tracking device, reliability and reproducibility of the tracking measures, software data processing of the acquired motion and finally precise measure of the relationship between the anatomical structures and the system acquisition points chosen.

The present study aimed to develop a new workflow for the use of the portable Polaris-NDI device and quantitatively validate the proposed operative workflow for the measurement of the mandibular range of motion in-vitro and in-vivo in a healthy sample using the developed acquisition technique.

\section{Materials and Methods}

\subsection{Subjects}

A convenient sample of five volunteers ( 4 males and 1 female aged 21 to 28 years, mean age $23.8 \pm 2.77$ years,) recruited among the students of the Department of Orthodontics of the University of Milan, Italy, participated in the study. The number of participants was kept low because the work was intended as a pilot study.

Inclusion criteria for the participants were the absence of TMJ problems according to the diagnostic criteria for Temporomandibular Disorders DC/TMD [20] with fully sound 
dentition. Subjects presenting any orthodontic devices were excluded. Participants were accurately informed about the study procedures and they were ensured that they could leave the study at any time. The study was conducted according to the guidelines of the Declaration of Helsinki, and approved by the Institutional Review Board of the University of Milan (protocol code IRB012020 doc SO 01).

\subsection{Experimental Set-Up}

For each subject, upper and lower dental impressions were taken and dental casts were obtained. A resin model of the vestibular inferior arch was designed to fit the inferior dental arch. Two copies of a rigid splint were designed using Catia v. R2019x (IBM, New York, NY, USA) and 3D-printed in resin for each volunteer from dental cast to fit the volunteer's buccal dental anatomy in a stable position (3D-Printed dental adapter) (Figure 1).

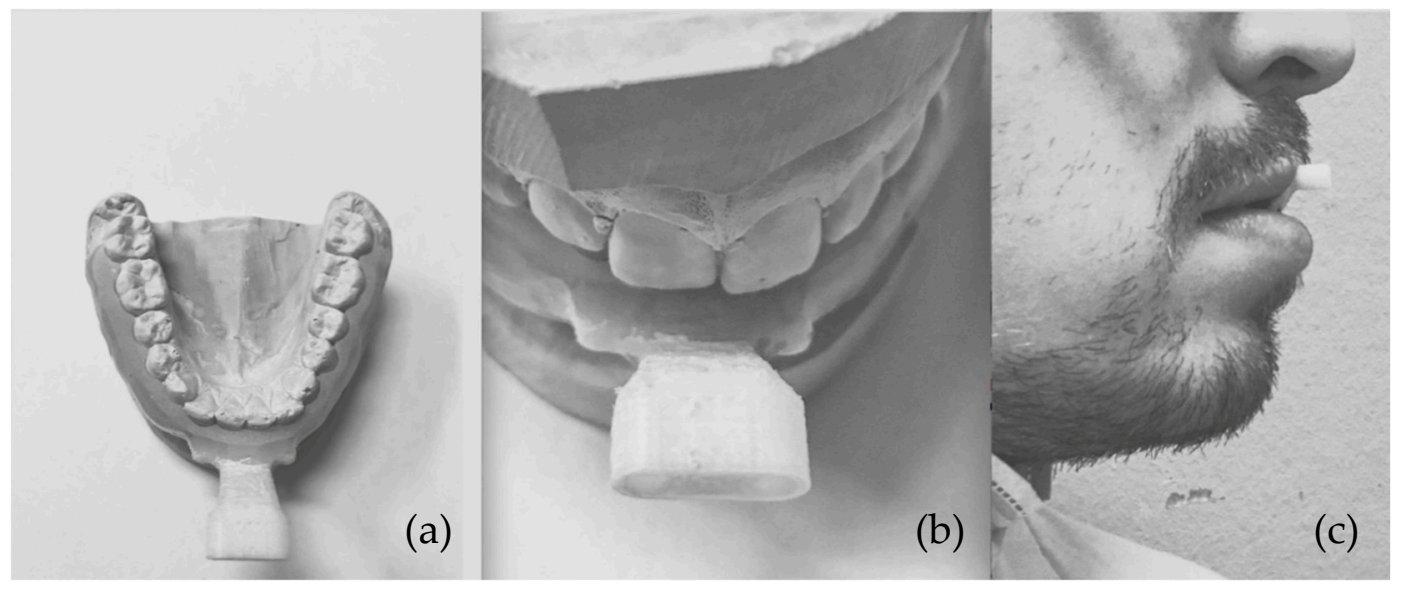

Figure 1. Dental resin model designed to fit the patient's inferior dental arch anatomy without interfering with occlusion. (a) Top view; (b) Detail of the connector for the standardized external support carrying the markers; (c) side view of the object in patient's mouth.

Four retro-reflective spherical markers (diameter $10 \mathrm{~mm}$ ) were attached with a distance of $30 \mathrm{~mm}$ from each other to a standardized external support. The external support was then connected by a metal connector to the 3D-printed dental adapter to form the Mandibular Tracking Support (MTS). Upon mounting of the MTS, the metal connector was scanned with a professional 3D structured-light scanner (inEos X5, Sirona, Charlotte, NC, USA) in order to determine the distance between the markers and the dental arch [21,22]. This geometrical relationship was used to derive the position of the teeth in the data analysis. Four additional retro-reflective markers were located on a standardized 3D-printed external set-up on the glabella (Cranial Tracking Support, CTS), well fixed to the head (Figure 2). The cranial support CTS defined a cranial plane of reference. The mandibular support MTS defined the movement plane and its movements were described relative to the cranial plane of reference.

Movements were recorded using a portable optoelectronic three-dimensional motion tracking system operating at $60 \mathrm{~Hz}$ (Northern Digital Inc., Polaris Spectra, Waterloo, ON, Canada). Briefly, the tracking device consists of two high-resolution infrared-sensitive charge-coupled-device video cameras coupled with a video processor with a working volume of $1500 \times 1300 \times 1450 \mathrm{~mm}^{3}(\mathrm{~W} \times \mathrm{H} \times \mathrm{L})$. A built-in software able to recognize the three-dimensional coordinates of the geometric center of up to eight passive markers during movement acquisition converted all the landmarks of each captured frame into a set of $x, y$, and $z$ spatial coordinates. The tracking device was placed on a stable tripod at a standard distance of $1.5 \mathrm{~m}$ from the location of the markers. Its orientation was set with a double spirit level and controlled between measurements. All metal items were removed from the field of view (FOV) of the device, including personal objects worn by the volunteers during the data acquisition (i.e., earrings or glasses). 


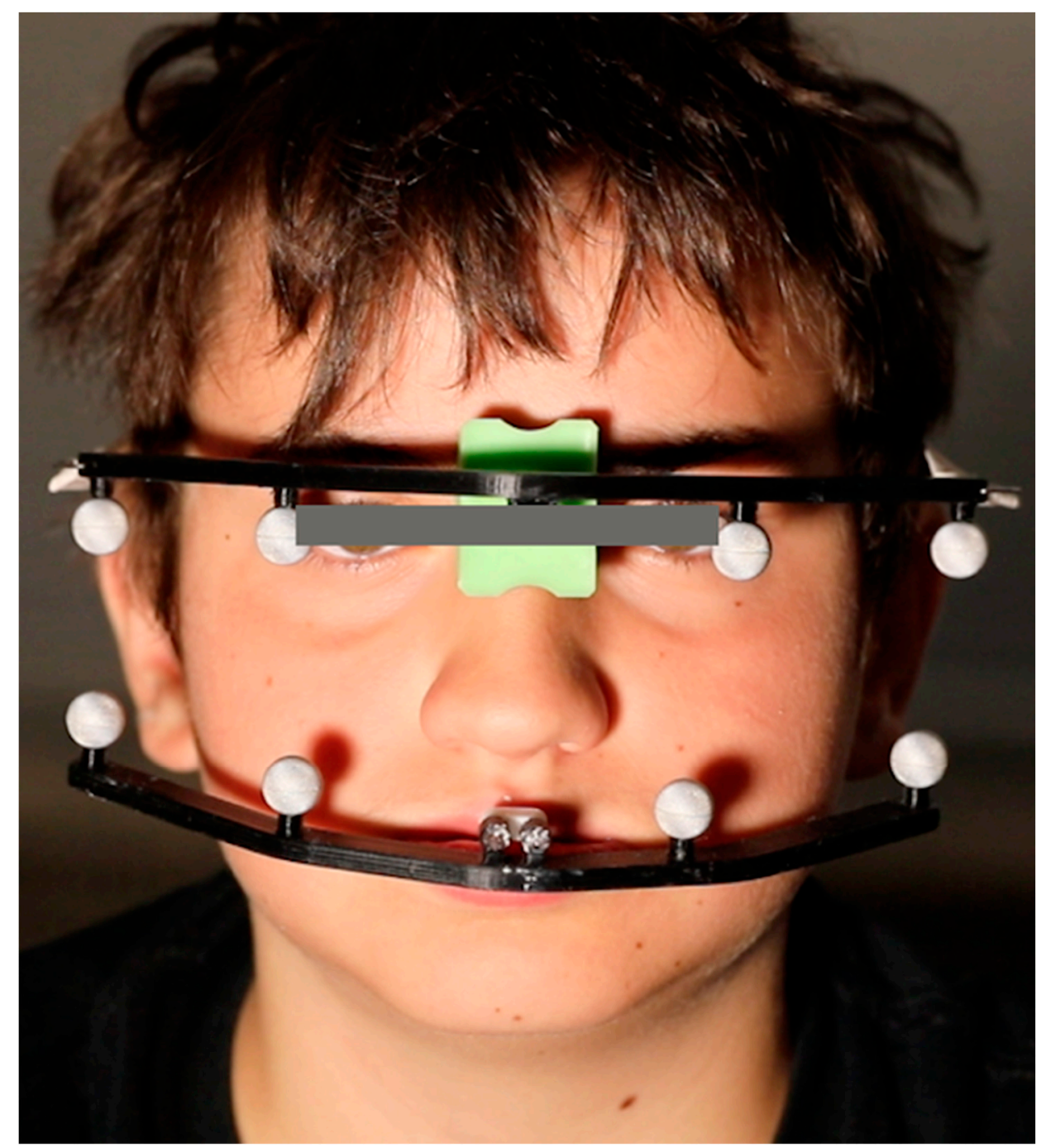

Figure 2. The upper and lower marker supports placed on the patient's head: the cranial support with reflective markers fits the nasion point with a soft body anteriorly and an elastic band posteriorly. The lower support fits the lower dental arch with a resin attachment and it is not interfering with occlusion.

\subsection{In Vitro Testing}

Before in vivo data collection on volunteer, the reproducibility and reliability of the system was tested with a series of in vitro recordings performed with prescribed movements using the same settings described above. The CTS and MTS were positioned on a rigid pedestal. The construct CTS was kept fixed on the support frame, whereas the MTS was positioned on a moving element and translated along the three main cartesian directions $(\mathrm{x}=$ left-right, $\mathrm{y}=\mathrm{up}$-down, $\mathrm{z}=$ back-front $)$ in a series of 10 repetitions for 10 times.

The maximum excursion vector of the MTS markers relative to the CTS markers was obtained and the measurement error calculated with respect to the prescribed mean value. Repetition variability was assessed by calculating the coefficients of variation (the percentage ratio of the standard deviation to mean) of the repetitions.

\subsection{In Vivo Data Collection}

At the time of the experimental session, each volunteer was given a brief explanation of the experimental protocol and was invited to perform the following movements: maximal mandibular excursions during the active opening, laterotrusion to the left and 
right, retrusion and protrusion with teeth in contact, keeping the head in a natural position. During laterotrusion, retrusion and protrusion, the volunteers were instructed to move the mandible at their best, forcing the limit for 2-3 s, to the right and the left, backward and forward while keeping the opposing teeth in contact [23]. After a few minutes of exercise to familiarize with the movements, the cranial tracking support was secured to the back of the head of the volunteer with an elastic band; the frontal point was located at the nasal point. The MTS was mounted in the subject's mouth without interfering with dental occlusion, to avoid disturbances in the movement performance, while keeping the wearing comfort as high as possible and allowing lip motion. The retention to the dental arch was secured form the 3D printed fitting of the device to the buccal surfaces of the teeth and their undercuts. If the fit and stability on the dental arches were not perfect, because for example insufficient undercuts to assure mechanical retention, few drops of fluid temporary composit resin (Fermit Ivoclar-Vivadent, Amherst, NY, USA) on the cutting-edge of the MTS were used to fix it to dental arches. The volunteer seated on a stool in a straight position facing the device cameras while wearing the marker supports [24] (Figure 3).

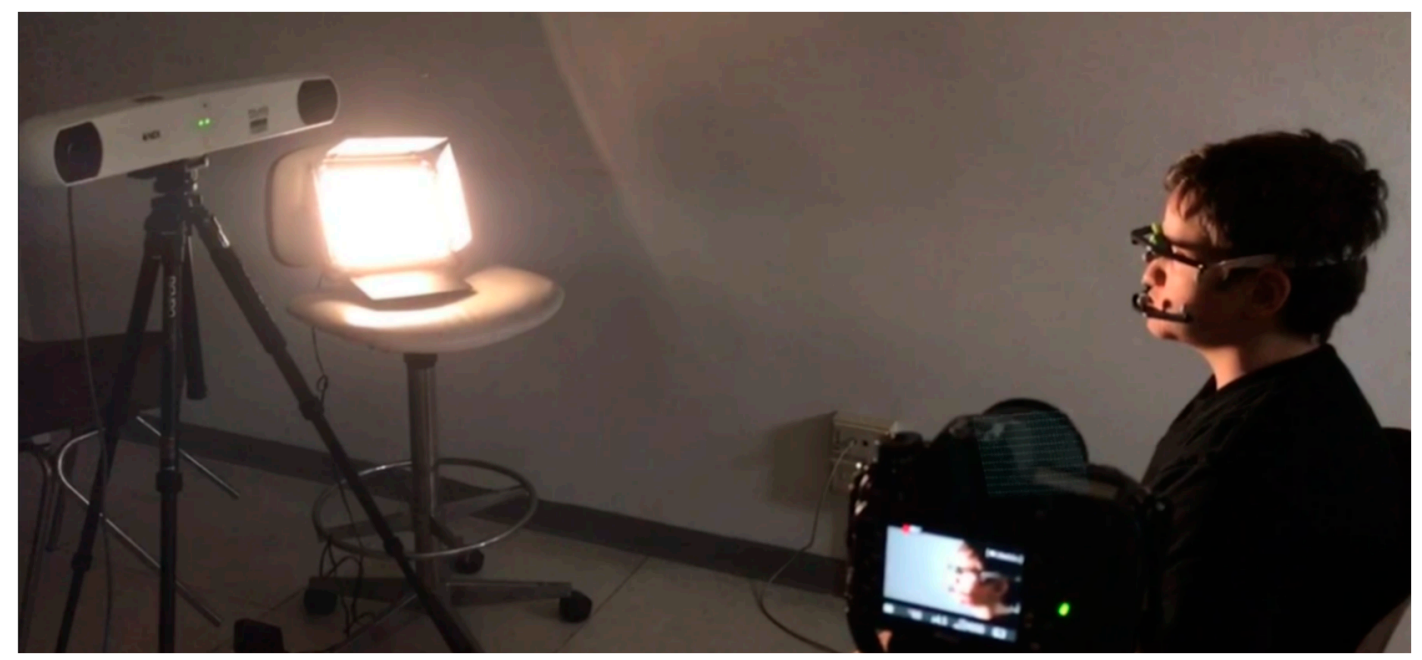

Figure 3. Acquisition set-up. The volunteer is sitting straight facing the cameras with the head in a neutral position and he is wearing the cranial and mandibular marker supports.

Each volunteer repeated the movements of the protocol three times during the recording session. The starting point for every movement $\left(\mathrm{T}_{0}\right)$ coincided with centric occlusion $\left(\mathrm{P}_{0}\right)$. Any difference with this starting point at the end of every cycle was discarded and required the repetition of the recording. For each volunteer, five separate sessions were repeated with a gap of at least $48 \mathrm{~h}$ between sessions. Every session was conducted by a different examiner. In each session, a new calibration of the instrument and new positioning of the markers were performed.

\subsection{Data Analysis and Statistics}

Spatial coordinates of the maximal excursion were manually identified on-screen by the operator recording the session, by marking the point, which coordinates are stored in the program and used for the further steps of the analysis. This operation leads to variability in the results of the session (Intra-operator reliability). An automatic detection method for the maximum excursion can be developed in future following examples available in the literature [13]. The position of the inter-incisal edge point (IEP) was derived from the position of the markers in space by knowing the relationship between the Mandibular Tracking Support (MTS) and the teeth, measured by the 3D-scan of the dental resin models with mounted external support. 
A dedicated custom-made algorithm automatically detected on-line the measurements $(\mathrm{mm})$ between the identified spatial coordinates of the lower central incisors inter-incisal edge point (IEP) at the reference limit points defined as:

1. The mandibular opening difference between IEP coordinates including vertical overbite during the active opening.

2. The maximum extent of the lateral mandibular movement difference between IEP coordinates during active lateral excursions (right and left).

3. The maximal retrusion difference between IEP during active retrusion.

4. The maximal protrusion difference between IEP during active retrusion corrected by including the horizontal overbite [22].

All measurements were relative to the cranial reference-the volunteer's head and neck movements were subtracted from the raw mandibular movement using three cranial markers and the reference zero point using an application of the original mathematical algorithms by Ferrario et al. [25].

For the calculation of the mandibular motion relative to the head, a series of mathematical transformations were applied to the collected data. The raw data were recorded in the fixed coordinate system $\mathrm{W}$, represented by the camera set-up. In this coordinate system, the head-related and mandible-related systems were defined by the spatial position of the Cranial Tracking Support CTS and the spatial position of the Mandibular Tracking Support MTS. The absolute movement of the head in the coordinate system of the cameras $\mathrm{W}$ was described by the transformation matrix ${ }^{\mathrm{W}} \mathrm{H}_{\mathrm{CTS}}$ and the absolute movement of the mandible was represented by the transformation matrix ${ }^{\mathrm{W}} \mathrm{H}_{\mathrm{MTS}}$. To obtain the relative motion of the mandible with respect to the head, the transformation (1) was applied to all the coordinates of the mandibular points.

$$
{ }^{\mathrm{CTS}} \mathrm{H}_{\mathrm{MTS}}=\left({ }^{\mathrm{W}} \mathrm{H}_{\mathrm{CTS}}\right)^{-1} \cdot{ }^{\mathrm{w}_{\mathrm{H}_{\mathrm{MTS}}}}
$$

The mandibular range of motion at the interincisor point was calculated starting from the maximum $y$ value for the mouth opening, $x$ values (right and left) for the maximum lateral movement, and $\mathrm{z}$ values for the retromandibular and protusive movements. Calculations were performed after $0.10 \mathrm{~mm}$ of movement as the precision of the instrument was set at $0.05 \mathrm{~mm}$.

\subsection{Analysis of Operator Reliability In Vivo}

To assess the method reliability, the measurements obtained during each in vivo experimental session were blindly analyzed by three different observers by reproducing the recordings in the original software in separate sessions. The sessions were compared by calculating an intraclass correlation coefficient (ICC) for each variable. For each subject, intra-session variability was assessed by calculating the coefficients of variation (the percentage ratio of the standard deviation to mean) of the three repetitions. For each volunteer, the three repetitions were averaged, and the mean values of the maximum mouth excursions, as expressed by the three-dimensional displacement of the mandibular interincisor point (in $\mathrm{mm}$ ) and its vertical projection (on the $y$-axis, in $\mathrm{mm}$ ) were calculated. Repeated sessions were compared by calculating an intraclass correlation coefficient (ICC) based on single-measurement, absolute-agreement, 2-way mixed-effects model for each variable. For each subject, intra-session variability was assessed by calculating the coefficients of variation (the percentage ratio of the standard deviation to mean) of the three repetitions. The level of significance was set at $5 \%$ for all comparisons $(p<0.05)$.

\section{Results}

\subsection{Reproducibility of In Vitro Tests}

The in vitro tests showed a mean error in the excursion vector of $0.45 \pm 0.28 \mathrm{~mm}$ in the left right direction, $0.54 \pm 0.26 \mathrm{~mm}$ in the back-forth direction and $0.42 \pm 0.29 \mathrm{~mm}$ in the up-down direction. The coefficient of variation was in the order of $10^{-3}$ for both 
left-right and back-forth directions, whereas it was one order of magnitude larger for the up-down direction (Table 1).

Table 1. Results of the in vitro reproducibility tests. The excursion error [mm] was used as a measure of the accuracy for the set-up; the coefficient of variation as a measure of its precision.

\begin{tabular}{ccc}
\hline Movement & Excursion Error $(\mathbf{m m})($ Mean \pm SD) & Coefficient of Variation (Mean \pm SD) \\
\hline left-right & $0.45 \pm 0.28$ & $0.002 \pm 0.001$ \\
back-forth & $0.54 \pm 0.26$ & $0.005 \pm 0.002$ \\
up-down & $0.42 \pm 0.29$ & $0.020 \pm 0.008$ \\
\hline
\end{tabular}

\subsection{Intra-Rater Reliability}

In order to determine in vivo the intra- and inter-rater variability, a total of 675 different motion recordings were analyzed. Overall ICC for each variable was excellent (ICC >0.90) except for retrusion which showed only good reliability level $($ ICC $=0.789)$. Total overall intra-rater ICC (all variables together) showed an excellent intra-rater reliability (ICC $=1.00$ with $95 \%$ confident interval $=1.00-1.00$ ) for 2 observes, and excellent reliability (ICC $=0.999$ with $95 \%$ confident interval $=0.999-1.00$ ) for third observer. Detailed results are presented in Table 2.

Table 2. ICC estimates for intra-rater reliability and their $95 \%$ confidence intervals.

\begin{tabular}{ccccccc}
\hline & \multicolumn{2}{c}{ Observer 1 } & \multicolumn{2}{c}{ Observer 2 } & \multicolumn{2}{c}{ Observer 3 } \\
\hline Movement & ICC & $\begin{array}{c}\mathbf{9 5 \% C I} \\
{[\text { Low-Up] }}\end{array}$ & ICC & $\begin{array}{c}\text { 95\%CI } \\
\text { [Low-Up] }\end{array}$ & ICC & $\begin{array}{c}\text { 95\%CI } \\
\text { [Low-Up] }\end{array}$ \\
\hline Maximal Opening & 0.999 & $0.998-1.000$ & 1.000 & $0.999-1.000$ & 0.994 & $0.985-0.998$ \\
Laterotrusion Right & 0.994 & $0.986-0.998$ & 0.994 & $0.985-0.998$ & 0.994 & $0.985-0.998$ \\
Laterotrusion Left & 0.996 & $0.991-0.999$ & 0.994 & $0.985-0.998$ & 0.994 & $0.985-0.998$ \\
Retrusion & 0.951 & $0.887-0.982$ & 0.994 & $0.985-0.998$. & 0.789 & $0.600-0.919$ \\
Protrusion & 0.994 & $0.986-0.998$ & 0.994 & $0.985-0.998$ & 0.984 & $0.963-0.994$ \\
\hline
\end{tabular}

\subsection{Inter-Rater Reliability}

Good inter-rater reliability (ICC > 0.75) was observed for all variables, except for retrusion which exhibited only moderate reliability $(\mathrm{ICC}=0.557)$. Detailed results are presented in Table 3.

Table 3. ICC estimates for inter-rater reliability of each variable and their $95 \%$ confidence intervals.

\begin{tabular}{ccc}
\hline Movement & ICC & 95\%CI \\
\hline Maximal Opening & 0.764 & $0.649-0.852$ \\
Laterotrusion Right & 0.779 & $0.666-0.863$ \\
Laterotrusion Right & 0.782 & $0.679-0.864$ \\
Retrusion & 0.557 & $0.390-0.705$ \\
Protrusion & 0.702 & $0.561-0.813$ \\
Maximal Opening & 0.764 & $0.649-0.852$ \\
\hline
\end{tabular}

\section{Discussion}

To authors' knowledge, this is the first study describing the reliability analysis of a mandibular kinematic tracking set-up developed using a simple, light, and portable hardware with infrared cameras and passive markers, and self-developed software to relate the mandibular motion to the craniofacial structures of the subject. The aim of the newly developed procedure was to obtain a method to calculate and mathematically exclude all supplementary movements of the head and neck from motion tracking. This was possible using a custom-designed algorithm that subtracted the movements from the superior anchored markers to the inferior markers in real-time. Similar transformation 
algorithms for the detection of relative mandibular motion were applied in the past by other authors by using a sophisticated custom-built tracking system based on active-markers and custom-made software [11].

The proposed workflow included 3D printing of custom-made adapters for the participants and 3D-scanning of the parts to obtain the geometrical relationship between the markers and the anatomy, allowing in short-time, implementation of an anatomically related tracking system. Previous studies showed that the precision and reliability of 3D scans and CAD techniques for digitalization of prosthodontic procedures was very high, although slightly dependent on the selected acquisition system [26,27].

Furthermore, the anchorage method played an important role in the registration of the movements. For the experiments, the shape of the arches was obtained from 3D digital scans of the dental cast models [21] and a resin model with a metal connection to the 3D printed connector was used in order to align with the anatomy of the inferior teeth of each volunteer. The use of a resin splint was also proposed with good results by Zhao et al. [14] with a full arch inferior resin splint including the lingual and vestibular surfaces, but this methodology might not apply to all patients. In contrast, Palla et al. [28] in their dynamic stereometry studies used metal clutches rigidly connected with dental polymers to the dental arches and an additional external face-bow carrying a reference for the anatomy registration. Calixtre et al. [29] described inter-rater reliability of a six-camera motion system with fifteen individual markers capturing at $120 \mathrm{~Hz}$. The use of a 3D-printed custom-fitting design, allowed the operator to avoid the use of dental polymers for the retention in most of the cases.

The precision of the NDI Polaris Spectra was tested in previous works [30], showing a maximal length error as $0.514 \mathrm{~mm}$ and a maximum clinical error, dependent on the markers configuration, of $1.3 \mathrm{~mm}$ during static recordings. In our in vitro tests, the mean error was calculated based on dynamic data, and gave comparable results as the maximal length error. As expected, the direction perpendicular to the cameras ( $\mathrm{z}$ or back-forth) gave the highest errors. Furthermore, the variability measured with the coefficient of variation was very low, in the order of $10^{-3}$.

The reproducibility of the method by different operators showed the highest precision (0.7-0.9) during simple movements as mouth opening and laterality, while the lowest values (0.5-0.7) were observed for retrusion. This was probably related to the difficulty of the movements and efforts of volunteers even after the training session. In comparison, Ferrario et al. [10] published ICC values between 0.57 and 0.76 using more complicated and non-portable instrumentation.

The overall simplicity of the proposed system was reported by all operators, also the volunteers did not report any discomfort or pain during the measurement sessions. The advantage of the system is that it could be rapidly applied to patients in different settings and conditions and in a near future used as a diagnostic tool in the TMD scenario, and also be used in dental offices in combination with $3 \mathrm{D}$ data to the $\mathrm{CBCT}$ and the patients (Figure 4). 


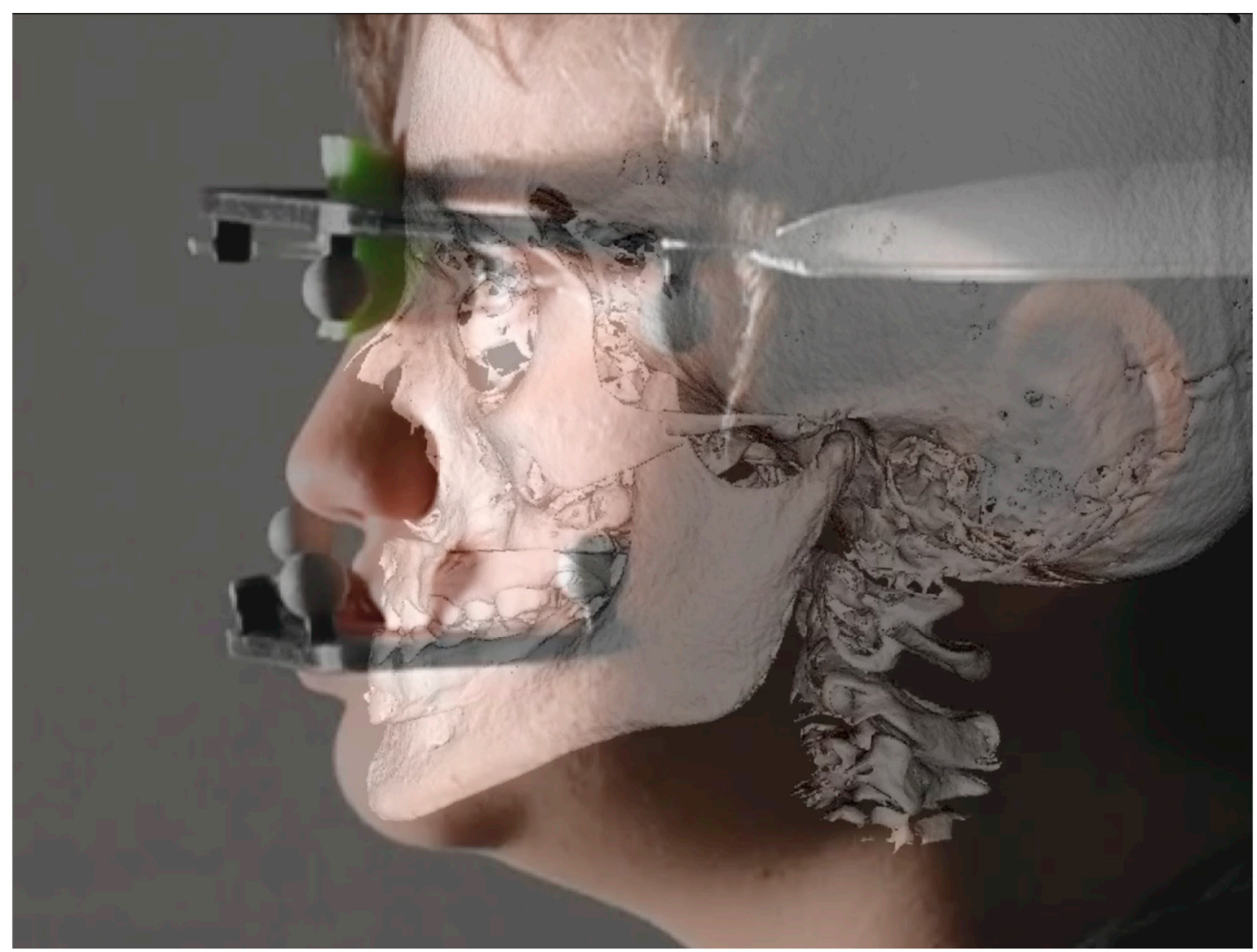

(a)

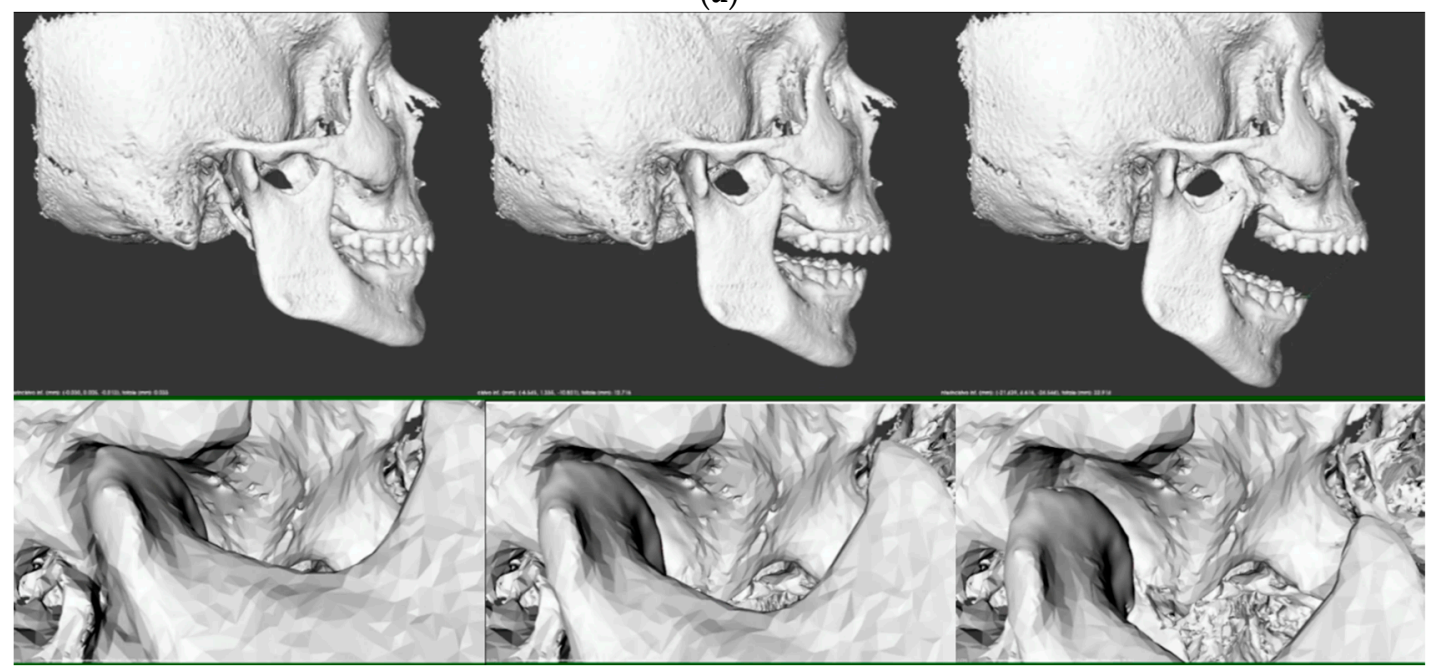

(b)

Figure 4. (a) The superimposition of the CBCT craniofacial structures with a photograph of the volunteer during the experimental session. (b) Combination of $\mathrm{CBCT}$ data with the mandibular kinematics recorded during the experiments. The figure shows the position of the condylar head of the mandible in closed (left), light open (middle) and maximal opening position (right).

The superimposition of CBCT and digital motion scans can be achieved by the use of the mucogingival junction as a reference as described by Ioshida et al. [31]. The visualization of the movement of the craniofacial structures avoids the necessity of performing more complicate and expensive exams as dynamic RMI and could offer a new method for gathering dynamic records from the patient in an era where full digitalization of the patient's records still represents a challenge [32]. 
The study was conducted in a small sample of healthy subjects under controlled conditions. Further data should be collected under different experimental conditions and involving dysfunctional patients in a wider population with better distribution considering the specific variables of the sample. Furthermore, gender- and age-related variables should be considered to avoid bias as described by other authors [33]. A technical limitation of the proposed system could be the difficulty to attach the connectors in patients with the absence or altered anatomy of the inferior teeth. Possible improvements of the system might include as well the development of an automatic detector for the maximal excursion position in order to exclude the intra-operator variability and increase the precision of the method. Based on these results further research is need to describe the variability range into different stomatognathic pathological conditions or mastication were the mandibular range of motion could be used as valuer or risk predictor of disease.

\section{Conclusions}

The study assessed a new method for the precise determination of relative mandibular movements by means of a portable motion-tracking device with a passive marker obtaining excellent results in terms of reliability for the majority of the performed measurements both in vitro and in vivo. This work opens the path to a digital workflow to be employed in clinical settings allowing the recording, analysis, and dynamic visualization of mandibular anatomy and kinematics by means of portable equipment.

Author Contributions: Conceptualization, G.M.T. and M.F.; methodology, G.M.T. and V.C.; data curation, M.F.; Formal analysis, V.C.; Investigation, M.F.; Supervision, G.M.T. and C.M.; Writingoriginal draft, V.C. and M.F.; Writing-review \& editing, V.C., G.M.T., C.M., L.M.G. and M.F. All authors have read and agreed to the published version of the manuscript.

Funding: This research received no external funding.

Institutional Review Board Statement: The study was conducted according to the guidelines of the Declaration of Helsinki, and approved by the Institutional Review Board of the University of Milan (protocol code IRB012020 doc SO 01).

Informed Consent Statement: Informed consent was obtained from all subjects involved in the study.

Data Availability Statement: All the data collected during the experiments and used for the analysis will be made available upon request.

Acknowledgments: The authors would like to thank A. Magni for his technical contributions and expertise in 3D printing methods.

Conflicts of Interest: The authors declare no conflict of interest.

\section{References}

1. Zago, M.; Luzzago, M.; Marangoni, T.; de Cecco, M.; Tarabini, M.; Galli, M. 3D Tracking of Human Motion Using Visual Skeletonization and Stereoscopic Vision. Front. Bioeng. Biotechnol. 2020, 8, 181. [CrossRef]

2. Furtado, D.A.; Pereira, A.A.; Andrade, A.d.O.; Bellomo, D.P.; da Silva, M.R. A specialized motion capture system for real-time analysis of mandibular movements using infrared cameras. Biomed. Eng. Online 2013, 12, 17. [CrossRef]

3. He, S.; Kau, C.H.; Liao, L.; Kinderknecht, K.; Ow, A.; Saleh, T.A. The use of a dynamic real-time jaw tracking device and cone beam computed tomography simulation. Ann. Maxillofac. Surg. 2016, 6, 113-119. [CrossRef]

4. Yoon, H.-J.; Zhao, K.D.; Rebellato, J.; An, K.-N.; Keller, E.E. Kinematic study of the mandible using an electromagnetic tracking device and custom dental appliance: Introducing a new technique. J. Biomech. 2006, 39, 2325-2330. [CrossRef]

5. Soboleva, U.; Laurina, L.; Slaidina, A. Jaw tracking devices-historical review of methods development. Part II. Stomatologija 2005, 7, 72-76.

6. Simon, S.R. Quantification of human motion: Gait analysis-benefits and limitations to its application to clinical problems. J. Biomech. 2004, 37, 1869-1880. [CrossRef]

7. Gordon, C.R.; Murphy, R.J.; Coon, D.; Basafa, E.; Otake, Y.; Al Rakan, M.; Rada, E.; Susarla, S.; Susarla, S.; Swanson, E.; et al. Preliminary development of a workstation for craniomaxillofacial surgical procedures: Introducing a computer-assisted planning and execution system. J. Craniofac. Surg. 2014, 25, 273-283. [CrossRef] 
8. Wiles, A.D.; Thompson, D.G.; Frantz, D.D. Accuracy assessment and interpretation for optical tracking systems. In Proceedings of the Medical Imaging 2004: Visualization, Image-Guided Procedures, and Display, Medical Imaging 2004, San Diego, CA, USA, 14 February 2004; Galloway, J.L., Ed.; SPIE: Bellingham, WA, USA, 2004; p. 421.

9. Throckmorton, G.S.; Talwar, R.M.; Ellis, E. Changes in masticatory patterns after bilateral fracture of the mandibular condylar process. J. Oral Maxillofac. Surg. 1999, 57, 500-508. [CrossRef]

10. Ferrario, V.F.; Sforza, C.; Lovecchio, N.; Mian, F. Quantification of translational and gliding components in human temporomandibular joint during mouth opening. Arch. Oral Biol. 2005, 50, 507-515. [CrossRef]

11. Gallo, L.M. Modeling of temporomandibular joint function using MRI and jaw-tracking technologies-mechanics. Cells Tissues Organs 2005, 180, 54-68. [CrossRef]

12. Leissner, O.; Maulén-Yáñez, M.; Meeder-Bella, W.; León-Morales, C.; Vergara-Bruna, E.; González-Arriagada, W.A. Assessment of mandibular kinematics values and its relevance for the diagnosis of temporomandibular joint disorders. J. Dent. Sci. 2021, 16, 241-248. [CrossRef] [PubMed]

13. Yuan, F.; Sui, H.; Li, Z.; Yang, H.; Lü, P.; Wang, Y.; Sun, Y. A Method of Three-Dimensional Recording of Mandibular Movement Based on Two-Dimensional Image Feature Extraction. PLoS ONE 2015, 10, e0137507. [CrossRef] [PubMed]

14. Zhao, T.; Yang, H.; Sui, H.; Salvi, S.S.; Wang, Y.; Sun, Y. Accuracy of a Real-Time, Computerized, Binocular, Three-Dimensional Trajectory-Tracking Device for Recording Functional Mandibular Movements. PLoS ONE 2016, 11, e0163934. [CrossRef]

15. Andrade, R.M.; Guimarães, L.R.; Ribeiro, A.P.; Pasqual Marques, A.; Crivello, O.; Gonçalves de Carvalho, B.K.; Amado João, S.M. Reliability in Mandibular Movement Evaluation Using Photogrammetry in Patients With Temporomandibular Disorders. J. Manip. Physiol. Ther. 2019, 42, 267-275. [CrossRef] [PubMed]

16. Mazzetto, M.O.; Anacleto, M.A.; Rodrigues, C.A.; Bragança, R.M.F.; Paiva, G.; Valencise Magri, L. Comparison of mandibular movements in TMD by means of a 3D ultrasonic system and digital caliper rule. Cranio 2017, 35, 46-51. [CrossRef]

17. Sójka, A.; Huber, J.; Kaczmarek, E.; Hędzelek, W. Evaluation of Mandibular Movement Functions Using Instrumental Ultrasound System. J. Prosthodont. 2017, 26, 123-128. [CrossRef]

18. Hwang, Y.-E.; Kang, S.-H.; Kim, H.-K. Errors according to the number of registered markers used in navigation-assisted surgery of the mandible. Head Face Med. 2019, 15, 6. [CrossRef]

19. Farronato, M.; Maspero, C.; Lanteri, V.; Fama, A.; Ferrati, F.; Pettenuzzo, A.; Farronato, D. Current state of the art in the use of augmented reality in dentistry: A systematic review of the literature. BMC Oral Health 2019, 19, 135. [CrossRef]

20. Schiffman, E.; Ohrbach, R.; Truelove, E.; Look, J.; Anderson, G.; Goulet, J.-P.; List, T.; Svensson, P.; Gonzalez, Y.; Lobbezoo, F.; et al. Diagnostic Criteria for Temporomandibular Disorders (DC/TMD) for Clinical and Research Applications: Recommendations of the International RDC/TMD Consortium Network* and Orofacial Pain Special Interest Groupt. J. Oral Facial Pain Headache 2014, 28, 6-27. [CrossRef]

21. Leonardi, R.; Lo Giudice, A.; Rugeri, M.; Muraglie, S.; Cordasco, G.; Barbato, E. Three-dimensional evaluation on digital casts of maxillary palatal size and morphology in patients with functional posterior crossbite. Eur. J. Orthod. 2018, 40, 556-562. [CrossRef]

22. Luz, J.G.; Uono, H.H.; Yamamoto, M.K. Passive mandibular border positions and their relationships with signs and symptoms of craniomandibular disorder. A study of linear and angular measurements. J. Nihon Univ. Sch. Dent. 1995, 37, 28-32. [CrossRef]

23. Ferrario, V.F.; Sforza, C.; Miani, A.; D'Addona, A.; Tartaglia, G. Statistical evaluation of some mandibular reference positions in normal young people. Int. J. Prosthodont. 1992, 5, 158-165. [PubMed]

24. Ferrario, V.F.; Sforza, C.; Tartaglia, G.; Barbini, E.; Michielon, G. New television technique for natural head and body posture analysis. Cranio 1995, 13, 247-255. [CrossRef] [PubMed]

25. Ferrario, V.F.; Sforza, C.; Schmitz, J.H.; Serrao, G. Comparison of unilateral chewing movements vs. dental guidance through the dental guidance ratio. J. Prosthet. Dent. 2001, 86, 586-591. [CrossRef]

26. Vafaee, F.; Firouz, F.; Mohajeri, M.; Hashemi, R.; Ghorbani Gholiabad, S. In vitro Comparison of the Accuracy (Precision and Trueness) of Seven Dental Scanners. J. Dent. (Shiraz) 2021, 22, 8-13. [CrossRef]

27. Lavorgna, L.; Cervino, G.; Fiorillo, L.; Di Leo, G.; Troiano, G.; Ortensi, M.; Galantucci, L.; Cicciù, M. Reliability of a Virtual Prosthodontic Project Realized through a 2D and 3D Photographic Acquisition: An Experimental Study on the Accuracy of Different Digital Systems. Int. J. Environ. Res. Public Health 2019, 16, 5139. [CrossRef] [PubMed]

28. Palla, S.; Gallo, L.M.; Gössi, D. Dynamic stereometry of the temporomandibular joint. Orthod. Craniofac. Res. 2003, 6 (Suppl. 1), 37-47. [CrossRef]

29. Calixtre, L.B.; Nakagawa, T.H.; Alburquerque-Sendín, F.; da Silva Grüninger, B.L.; de Sena Rosa, L.R.; Oliveira, A.B. Inter- and intra-rater reliability of 3D kinematics during maximum mouth opening of asymptomatic subjects. J. Biomech. 2017, 64, 245-252. [CrossRef]

30. Elfring, R.; de La Fuente, M.; Radermacher, K. Assessment of optical localizer accuracy for computer aided surgery systems. Comput. Aided Surg. 2010, 15, 1-12. [CrossRef] [PubMed]

31. Ioshida, M.; Muñoz, B.A.; Rios, H.; Cevidanes, L.; Aristizabal, J.F.; Rey, D.; Kim-Berman, H.; Yatabe, M.; Benavides, E.; Alvarez, M.A.; et al. Accuracy and reliability of mandibular digital model registration with use of the mucogingival junction as the reference. Oral Surg. Oral Med. Oral Pathol. Oral Radiol. 2019, 127, 351-360. [CrossRef] 
32. Mangano, C.; Luongo, F.; Migliario, M.; Mortellaro, C.; Mangano, F.G. Combining Intraoral Scans, Cone Beam Computed Tomography and Face Scans: The Virtual Patient. J. Craniofac. Surg. 2018, 29, 2241-2246. [CrossRef] [PubMed]

33. Mapelli, A.; Galante, D.; Lovecchio, N.; Sforza, C.; Ferrario, V.F. Translation and rotation movements of the mandible during mouth opening and closing. Clin. Anat. 2009, 22, 311-318. [CrossRef] 